\title{
FMH Consulting Services - dal 1.12.2014 anche in Ticino
}

Dal $1^{\circ}$ dicembre 2014, FMH Consulting Services, specializzato nei servizi per medici e studi medici, offre le sue prestazioni anche in Ticino. L'ampia varietà di servizi comprende fra gli altri:

- Analisi dell'ubicazione dello studio medico

- Pianificazione e allestimento dello studio medico

- Consigli relativi agli studi medici associati

- Pianificazione e gestione della successione

- Calcolo del valore di cessione globale / vendita dello studio medico

- Cessione o rilevamento dello studio medico

Il nuovo partner FMH Consulting Services in Ticino, la società SAC Studio Assistenza Commerciale SA, dispone di una grande esperienza nel settore medicale. Fondata nel 1985, SAC offre già da 20 anni, tramite FMH Fiduciaria Services, una vasta gamma di servizi nel settore fiduciario che vanno dalla gestione societaria, al controllo di gestione, alla consulenza fiscale e aziendale, alla revisione.

La società si occupa con professionalità della gestione di studi medici; gli anni di esperienza hanno permesso la creazione di una solida formazione e di un importante bagaglio di nozioni, che vengono quotidianamente messe a disposizione dei clienti medici per qualsiasi tipo di necessità in ambito economico-finanziario e per tutto ciò che attiene alla gestione del loro studio medico.

Le competenze acquisite e il personale, qualificato e professionale, sono aspetti che da sempre contraddistinguono la società e che permettono di offrire il massimo dell'efficienza, garantendo lo sviluppo di un rapporto di fiducia verso i clienti e le varie istituzioni, nella gestione dei mandati FMH Services affidati.
Grazie ai molteplici servizi messi a disposizione ed alla nostra esperienza, i professionisti medici hanno tutti gli strumenti utili per rendere efficiente e prospero il proprio studio medico.

SAC Studio Assistenza Commerciale SA FMH Consulting Services

Corso San Gottardo 89

6830 Chiasso

Tel. 0916976080 - Fax 0916836826

www.fmhconsulting.ch

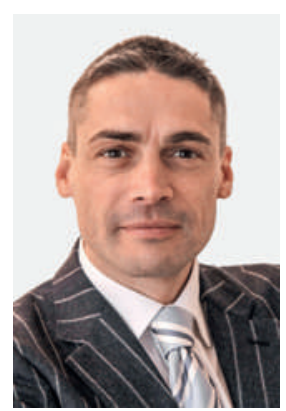

Franco Pozzi

Fiduciario con Attestato Professionale Federale

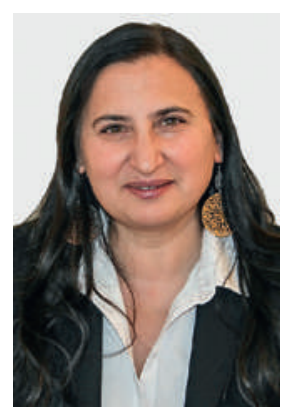

Rosa Giannetta

Laureata in Economia e Commercio 